\title{
émulations
}

\section{Les mécaniciens de rue entre précarité et autoréalisation}

\section{Assujettissement aux incertitudes et subjectivation en tant que professionnel}

\section{Denis Giordano}

Émulations - Revue de sciences sociales

2018, n²8, «Précarité, précaires, précariat. Allers-retours internationaux ».

\section{Article disponible à l'adresse suivante}

https://ojs.uclouvain.be/index.php/emulations/article/view/giordano

\section{Pour citer cet article}

Denis Giordano, « Les mécaniciens de rue entre précarité et autoréalisation. Assujettissement aux incertitudes et subjectivation en tant que professionnel », Émulations, $\mathrm{n}^{\circ} 28$, Mise en ligne le 20 février 2019. DOI : 10.14428/emulations.028.07

Distribution électronique : Université catholique de Louvain (Belgique) : ojs.uclouvain.be

(C) Cet article est mis à disposition selon les termes de la Licence Creative Commons Attribution, Pas d'Utilisation Commerciale 4.0 International. http://creativecommons.org/licenses/by-nc/4.0/

Éditeur : Émulations - Revue de sciences sociales / Presses universitaires de Louvain https://ojs.uclouvain.be/index.php/emulations

ISSN électronique : 1784-5734

PUL PRESSES 


\title{
Les mécaniciens de rue entre précarité et autoréalisation
}

\author{
Assujettissement aux incertitudes et \\ subjectivation en tant que professionnel
}

Denis Giordano ${ }^{1}$

\begin{abstract}
[Résumé] Dans cette contribution, j'explore les tensions entre la précarité comme source d'assujettissement aux incertitudes de la situation sociale et économique contemporaine et la précarité comme terrain où l'on rencontre des modalités spécifiques de subjectivation. Mon intérêt s'est focalisé sur la figure du mécanicien de rue qui réalise des activités de réparation sans avoir de licence. Tout en se confrontant avec une situation de forte précarité et de nombreuses difficultés, ces mécaniciens peuvent se construire une réputation comme professionnels de la réparation. Ce processus contribue à la construction de leur identité et influence leur rôle d'acteur dans les quartiers où ils vivent et travaillent. Toutefois leur pratique, ainsi que les solutions créatives adoptées tout au long du travail, ne leur permettent pas de s'émanciper de la situation d'incertitude où ils agissent et à laquelle sont assujettis.

Mots-clés : mécanicien de rue, précarité, identité professionnelle, banlieue parisienne, bricolage

[Abstract] This article explores the tensions that emerge between precarity as a form of subjugation to the conditions of social and economic uncertainty and precarity as a field within which we can find particular modalities of subjectivation. My focus is on street mechanics: people who fix cars without a licence to do so. This social group faces a high level of precarity and a number of difficulties, whilst at the same being able to build a reputation as professionals in their field. This process contributes to the scaffolding of their identity and influences their role of social actors in the neighborhoods they inhabit. However, their practice and their creativity does not permit them to overcome the uncertain conditions of their life and work.
\end{abstract}

Keywords: street mechanics, precarity, self-realization, Paris suburbs, bricolage

\section{Introduction}

Dans cette contribution, j'explore les tensions entre la précarité comme source d'assujettissement aux incertitudes de la situation sociale et économique

\footnotetext{
${ }^{1}$ COMPTRASEC, Université de Bordeaux/LCSP, Université Paris Diderot.
} 
contemporaine (Standing, 2011) et la précarité comme terrain où l'on rencontre des modalités spécifiques de subjectivation (Armano, Murgia, 2012 ; Berardi, 2016).

Mon intérêt s'est focalisé sur une figure spécifique de travailleur, le mécanicien de rue, qui réalise des activités de réparation de véhicules sans avoir de licence, avec un outillage limité, en s'installant dans des espaces publics ou privés sans autorisation. Cette activité fait partie du monde de l'économie informelle (Portes, Castells, Benton, 1989 ; Coletto, 2010) qui se manifeste au sein des pays européens (Williams, 2013) et peut être considérée comme une forme de travail précaire marquée par une forte instabilité et imprévisibilité (Bouffartigue, Busso, 2013). On se confronte donc à une forme de précarité qui ne se définit pas à partir d'un contrat de travail (qui demeure absent) mais qui s'explicite à travers les conditions de travail et à partir de l'expérience subjective (Cingolani, 2006) vécue par les mécaniciens de rue.

L'étude de cas de ces travailleurs permet de se confronter à leur expérience quotidienne composée d'un assujettissement à des conditions de précarité qui, en même temps et grâce à la possibilité de réalisations de services aux clients, se montre aussi comme un terrain de subjectivation et de construction de l'identité professionnelle. J'expliciterai cette problématique en mobilisant les réflexions élaborées à partir d'une recherche qui s'est attachée à explorer le monde social de ces travailleurs installés dans les quartiers populaires de la banlieue parisienne.

Bien qu'inscrite dans l'économie informelle, la mécanique de rue est loin d'être une activité réalisée de manière clandestine par des individus isolés. Elle se montre profondément dépendante de la réalité sociale des quartiers et des espaces urbains où elle se déroule. L'acceptation des mécaniciens de rue est surtout le fait des habitants de ces quartiers qui se confrontent également à des difficultés majeures pour trouver des emplois stables ainsi qu'à une continuité de revenu (Fol, 2010).

Pour comprendre les activités du mécanicien de rue, il faut considérer, au-delà de ses pratiques les plus opérationnelles de travail, son univers relationnel, composé par les liens avec ses voisins et « collègues » (Giordano, 2016). Pour les mécaniciens, la maîtrise de «l'art de la débrouille» devient un moyen crucial pour s'identifier comme « experts » et un élément capable de concourir à la construction de leur réputation.

Je donnerai, d'abord, quelques éléments sur la démarche méthodologique suivie. Dans une partie analytique, ensuite, je me focaliserai sur les formes d'action des mécaniciens qui illustrent leur horizon quotidien entre précarité et expression d'une compétence spécifique. En conclusion, enfin, je formulerai des considérations plus théoriques à partir de leur expérience et de leur manière de se considérer comme des experts et des professionnels de la réparation en dépit de leur activité informelle.

\section{Méthodologie}

La recherche est issue d'un travail de terrain de dix-huit mois réalisé lors de ma thèse, dans une commune de la Seine-Saint-Denis que l'on appellera Montaigne. À travers une posture vouée à la participation et à l'action au-delà de la simple observation 
ethnographique (Wacquant, 2002), j'ai acquis une connaissance directe de l'activité des mécaniciens de rue agissant dans ces quartiers, en m'engageant (Jounin, 2008) dans les réparations. Cette démarche s'est concentrée sur les pratiques de travail des mécaniciens en considérant attentivement l'univers matériel et situationnel où elles se déroulent (Corbin, Strauss, 1993; Schatzki, Knorr Cetina, von Savigny, 2001 ; Bruni, Gherardi, 2007).

Après une première phase, basée sur l'observation systématique de plusieurs mécaniciens de rue et face aux difficultés à développer des liens de confiance, j’ai pris la décision de m'engager directement à leurs côtés, en proposant mon aide pour les réparations en suivant une approche de participation observante (Bastien, 2007). L'engagement direct du corps du chercheur dans les activités a ainsi été utilisé comme moyen supplémentaire de connaissance (Pink, 2009; Pillon, 2012). De cette façon j'ai pu mettre les « mains dans le cambouis » avec les mécaniciens, en partageant certaines de leurs activités, du temps de travail dans la rue et un peu de leur expérience quotidienne. En particulier, pendant six mois, j'ai accompagné le travail de trois d'entre eux. $\mathrm{Mel}^{2}, 58$ ans (2013), avec qui j'ai passé le plus de temps et qui faisait également quelques missions ponctuelles en tant que brancardier dans un hôpital parisien; Guy, 41 ans, ancien garagiste au chômage ; Daimou, 23 ans et sans papier qui vivait des réparations qu'il arrivait à réaliser ${ }^{3}$. Pour les trois, l'argent des réparations constitue une partie indispensable de leur revenu, souvent menacée par les aléas de leurs recettes.

J'ai été attentif à l'expression de leur savoir-faire en écoutant les mots accompagnant les satisfactions ou les déceptions ainsi que les considérations au sujet de leur rôle dans le monde des réparations. Pour mieux comprendre la place des mécaniciens de rue dans les quartiers où ils agissent, ainsi que les spécificités de leur activité, j’ai effectué une observation participante dans trois garages de Montaigne ${ }^{4}$. Il s'agissait ainsi de mieux saisir les formes innovantes de service proposées par les mécaniciens de rue et d'enquêter sur leur considération par les professionnels « officiels».

\section{Mécaniciens de rue et pratiques de bricolage}

Montaigne, commune de la proche banlieue parisienne, présente plusieurs quartiers populaires qui sont les plus éloignés du métro et du centre-ville historique de la commune. La position géographique de ces quartiers périphériques joue un rôle dans l'importance du véhicule, mais aussi dans la surreprésentation de chômeurs et de travailleurs précaires parmi les habitants (Sassen, 2001 ; Clerval, 2013 ; Rosa Bonheur,

\footnotetext{
${ }^{2}$ Pseudonyme, comme les prénoms suivants.

${ }^{3}$ Même au-delà de ces trois, les mécaniciens rencontrés étaient tous des hommes, ce qui confirme la dimension masculine du monde de la réparation automobile (Weber, 1989).

${ }^{4}$ Cette enquête supplémentaire a eu une durée d'une semaine de travail dans chacun des trois garages.
} 
2014). Un tel contexte aide à comprendre d'emblée la présence ainsi que la sollicitation diffuse des mécaniciens de rue pour intervenir sur les problèmes liés aux véhicules. Un autre élément préliminaire à prendre en compte concerne la nature informelle de leur activité: ils ne disposent pas d'autorisation, ni de garage, et leur action peut être sanctionnée à tout moment par les différentes formes d'autorité présentes sur les lieux (la police, les gardiens des HLM, les riverains dérangés). Cette condition pose un obstacle en permanence présent à l'horizon de l'exercice de leur travail.

Les pratiques des mécaniciens de rue révèlent une condition de forte précarité à laquelle ils sont exposés, en particulier dans la perspective temporelle incertaine et dans l'exposition à tout type d'imprévu. Tout d'abord, leur univers instrumental est approximatif et bien loin d'un standard approprié pour réaliser l'ensemble des réparations: au manque d'outillage technique s'ajoute la vétusté d'instruments et d'outils le plus souvent non adaptés. Un élément saute aux yeux : pour soulever les véhicules ils utilisent le simple cric "de base» et des béquilles en acier comme protection, au lieu de bénéficier des ponts élévateurs présents dans les garages. Il faut donc souligner la dangerosité et les risques auxquels les mécaniciens sont exposés en agissant dans la rue. Ils essayent de se protéger autant que possible car leur corps, soumis à de nombreux dangers, est considéré comme un capital fragile à préserver.

Les risques les plus évidents sont liés à la sécurité du travail et aux possibilités d'accident. Il faut ajouter à cela les risques et les conséquences liés à une certaine pénibilité du travail à l'extérieur, comme j'ai eu occasion de l'éprouver. En travaillant dehors et le plus souvent à même le sol, on a froid et on combat des ennemis aussi insidieux que le vent ou l'humidité. Le mal de dos est un fidèle compagnon de route; deux des trois mécaniciens avec qui j'ai travaillé en étaient affligés, avec parfois des douleurs les obligeant à s'arrêter, bien évidemment sans aucune indemnité :

Avec mon dos maintenant... je cherche seulement de faire des choses rapides : s'il y a des freins à changer ou des choses comme ça. Mais si je reste allongé par terre à faire des efforts... après je n'arrive plus à me relever ! [...] J'ai la sciatique merde... (Mel, carnet de terrain, 4/12/2011)

Leur activité quotidienne s'exerce dans un équilibre précaire construit à la fois dans et par l'acceptation - temporaire et conditionnée - des riverains, grâce à la confiance (ou l'espoir) que la police n'interviendra pas, et surtout en mobilisant toutes leurs compétences, manuelles et relationnelles, pour réaliser les réparations. La continuité d'exercice dérive de la capacité du mécanicien de rue à entretenir un cercle de personnes ayant confiance dans son action et qui, grâce au « bouche à oreille », peut assurer régulièrement une clientèle.

Face à ces incertitudes, s'avère cruciale la capacité des mécaniciens à jongler avec tout type de ressources à leur disposition, en pratiquant une démarche que je qualifie de « débrouille créative ». Pour réaliser ses réparations dans la rue, le mécanicien doit à chaque fois réunir des ressources suffisantes, s'arranger donc avec les «moyens de bord »(Lévi-Strauss, 1961 : 31) : avoir accès à un lieu où il pourra travailler, disposer d'un 
outillage suffisant, mobiliser les compétences pour conduire l'intervention. Ces pratiques de composition et recomposition incessante des ressources - matérielles et relationnelles - constituent l'horizon d'action quotidienne des mécaniciens, pleinement inscrits parmi les "bricoleurs », en suivant les analyses de Lévi-Strauss (1961) et Sennett (2008). Cette conception du bricolage associe la capacité à trouver des solutions appropriées et innovantes avec les limites et les obstacles présents dans la situation. Le bricoleur doit exprimer ses compétences dans la "débrouille» en se contentant des possibilités à sa disposition tout en démontrant son habileté à l'intérieur même des contraintes.

Bien que s'exerçant hors du cadre de la loi, les difficultés et les risques associés à l'activité de mécanicien de rue peuvent se retrouver également dans une multiplicité de métiers propres au précariat contemporain, en particulier en termes de sécurité d'emploi et de perspective professionnelle (Standing, 2011). Pratiquer le bricolage se révèle aujourd'hui être une compétence importante pour exercer une multiplicité d'autres métiers marqués par une incitation croissante à la multiactivité, à la responsabilité et à l'autonomie.

\section{Maîtriser l’art de la débrouille}

Se référer aux mécaniciens de rue comme à des «bricoleurs » dérive aussi des mots rencontrés sur le terrain, prononcés par les garagistes ou par leurs clients, comme Didier (20/04/2011) :

Eux sont des bricoleurs, ils ne sont pas des garagistes non plus. [...] Quand ils doivent réparer une voiture, ils ont l'objectif d'atteindre un niveau minimal : si la bagnole marche c'est ok pour eux, c'est bien comme ça...

La définition de Didier manifeste la distinction entre les mécaniciens et les professionnels « officiels » de la réparation - les garagistes - en soulignant en même temps le style spécifique appliqué à la réparation par les mécaniciens de rue. Leur objectif est différent de celui des garagistes : la voiture ne doit pas respecter tous les critères officiels, il suffit qu'elle soit à nouveau en état de marche.

Pour arriver à ce résultat, certes d'un niveau plus modeste par rapport aux possibilités d'un professionnel, mais déjà suffisant pour les clients qui demandent l'intervention, le mécanicien de rue active une multiplicité de formes de bricolage :

- se satisfaire d'un nombre limité d'outils et de pièces, parfois non-professionnels ;

- avancer par tâtonnements, en essayant ;

- mobiliser des connaissances pour offrir des services variés.

Ces pratiques de bricolage s'expriment en se confrontant à la dimension matérielle des pratiques de travail, mais aussi à leur dimension relationnelle : en mobilisant toute une série de connaissances, liens et relations avec d'autres acteurs du terrain. Du point de vue de la planification du travail, les mécaniciens de rue doivent être capables d'« organiser l'immédiat » et de " minimiser l'intervention » lors de leurs missions. Le mécanicien ne peut pas appliquer un protocole fixe et doit « commencer de zéro » à 
chaque fois : à la fois en s'accordant avec le client (sur le type, le prix et le temps de l'intervention) et en vérifiant rapidement les outils à sa disposition. Il est indispensable de réduire le plus possible les opérations à prévoir à cause de leurs limites techniques. L'organisation de l'immédiat souligne un rapport de normalisation de l'imprévu, élément récurrent et ordinaire du travail des mécaniciens de rue. S’ils ne peuvent pas imiter les organisations plus structurées, tels que les garages, ils doivent se montrer extrêmement flexibles et réactifs pour trouver ou inventer des solutions aux difficultés et réussir à s'adapter aux contingences pour parvenir au résultat.

À de nombreuses occasions, j'ai assisté aux échanges entre les mécaniciens et d'autres acteurs de la réparation automobile: garagistes, gérants de casses auto, opérateurs du contrôle technique, autres mécaniciens de rue. Ces échanges permettent aux mécaniciens de récupérer des pièces, des outils ou de la technologie (les valises électroniques par exemple) nécessaires pour leurs réparations. Les limites dans la disponibilité des ressources imposent au mécanicien de pratiquer un style de réparation voué à minimiser son intervention. On parle de style car c'est une démarche propre et véritable de positionnement sur le marché de la réparation. Pendant que les garagistes - lors des réparations - doivent respecter des critères liés aux standards complexes de sécurité, les mécaniciens de rue - en considérant aussi la nature de leur activité - n'ont pas les mêmes obligations.

Minimiser l'intervention signifie aussi de limiter tout changement de systèmes ou de pièces dans les véhicules, tout en acceptant de récupérer et/ou d'installer des pièces de rechange provenant des casses automobiles. La réduction a minima du besoin de main d'œuvre et de substitution des pièces peut conjuguer les nécessités des deux parties engagées dans la réparation de rue : les mécaniciens, soucieux de limiter leur effort ; les clients, qui peuvent ainsi bénéficier de prix plus favorables. Ces derniers peuvent être très attractifs car la rétribution ${ }^{5}$ des mécaniciens de rue est basse et le choix de limiter le changement de pièces contribue aussi à la réduction des frais. En effet, souvent, chez les garagistes, un système (freins, transmission...) doit être changé en entier lors d'une panne ce qui occasionne des frais élevés, alors que chez les mécaniciens de rue, le changement concerne souvent une seule pièce spécifique avec un résultat plus économique, bien que moins sûr.

La situation décrite montre qu'il existe une demande de service de réparation capable de remettre en état de marche un véhicule en dépensant le minimum d'argent. Cette volonté, associée aux risques de sécurité et au manque de manutention de la voiture, décrit le rôle occupé par les mécaniciens de rue dans le marché de la réparation. Ils ne se positionnent pas (seulement) comme des concurrents à prix dérisoires des

\footnotetext{
${ }^{5}$ Souvent, les mécaniciens de rue se font payer avec une formule au forfait pour des réparations précises (Mel et Guy parmi eux). Si les garagistes demandent entre 30 et $50 €$ l'heure, les mécaniciens de rue que j'ai rencontrés touchent en général entre 10 et $20 €$ l'heure quand ils appliquent cette formule (Daimou et parfois Mel lors des réparations les plus simples).
} 
garagistes, mais acceptent d'entamer une réparation dans des conditions spécifiques où la réduction des frais est souvent un impératif. Il faut enfin rappeler que parfois les garagistes refusent de réparer certains véhicules jugés trop vétustes ${ }^{6}$.

Suite à mon expérience dans les quartiers de l'enquête, je n'ai pas observé d'antagonisme ouvert entre garagistes et mécaniciens de rue, comme signalé dans d'autres situations (Rosa Bonheur, 2017). Il existe un positionnement hiérarchique clair sur le marché de la réparation : garagistes et mécaniciens de rue ne font pas le même métier, ce que traduisent les mots de Dino, un garagiste (15/05/12) :

$\mathrm{Au}$ final eux [les mécaniciens de rue] travaillent à leur sauce... en se débrouillant. Et les gens qui vont chez eux c'est exactement ça qu'ils cherchent. Ici nous faisons les réparations nickels quoi...

Les réparations, surtout celles des véhicules marqués par l'usure ou ayant de nombreux problèmes, peuvent en l'occurrence devenir le patrimoine privilégié des mécaniciens de rue. Ils détiennent ce patrimoine et il leur est accordé (comme le montre l'extrait précédent) une légitimité à s'occuper du «sale boulot » des garagistes (Hughes, 1951, 1959) en demeurant en bas de l'échelle des réparations. Mais, grâce à la légitimité qui leur est accordée par d'autres professionnels et par les clients, les mécaniciens de rue peuvent aussi se sentir comme une partie légitime du monde des professionnels de la réparation.

\section{Devenir un expert et se faire reconnaître comme tel}

Les mécaniciens proposent aux clients un ensemble de services qui vont bien au-delà des seules réparations. Ils apportent leur aide lors de l'achat ou la cession d'un véhicule, se chargent d'un déplacement en cas de panne improvisée ou pour les contrôles techniques, ainsi que pour la « chasse » aux pièces de rechange dans les casses auto.

C'est grâce au tissu de relations entretenu avec les clients, les fournisseurs et les collègues (formels ou non), qui fait toujours l'objet d'un équilibre instable, que les mécaniciens de rue continuent à réaliser leurs services de réparation. Cette démarche confirme leur inscription dans une niche du marché de la réparation (Castel, 2007), où ils occupent un segment professionnel spécifique et proposent des formes caractéristiques de service. D’abord, ils multiplient les efforts pour aller de manière flexible à la rencontre de leur clientèle : ils se déplacent suite à un appel téléphonique pour des réparations là où le véhicule est en panne; ils se proposent pour des expertises ; ils offrent des arrangements pour contourner des règles (par exemple lors de contrôles techniques).

Les mécaniciens deviennent des repères faciles et accessibles en réalisant des réparations en présence du client. Leur organisation très sommaire les oblige à réparer

${ }^{6}$ Comme exprimé explicitement par un garagiste : « Oh, avec ta camionnette, change-la ! Tu vois? Tous les mois tu passes ici, tu peux pas continuer comme ça. Cherche des occasions! C'est la dernière fois que je veux voir ce tas de ferraille ici, d'accord ? » (César, 03/05/2012). 
à flux tendu : le client assiste à la réparation et ainsi ne se sépare jamais de sa voiture. Ils proposent des services «immédiats» particulièrement appréciés par les clients, surtout lors de déplacements suite à une panne, en limitant ainsi leur impossibilité de planifier des réparations par manque d'espace et d'organisation.

Grâce à la confiance manifestée par les clients qui les sollicitent, les mécaniciens se voient reconnus comme des experts dans la réparation ${ }^{7}$. Établir des relations stables avec ses clients est une source d'opportunités économiques en plus de la gratification symbolique liée à la reconnaissance de son propre rôle de professionnel. Les mécaniciens qu'on a rencontrés ont souvent manifesté leur volonté de se créer et de garder « un cercle d'habitués », soit pour des raisons de discrétion, soit pour continuer à suivre des véhicules et ainsi démontrer leur habileté. Être reconnus comme professionnels est une puissante source de satisfaction lors de l'exercice d'une activité (Garfinkel, 1963) : la reconnaissance sociale est prioritaire dans un contexte informel, où les garanties sont liées aux accords verbaux et aux mains serrées (Laé, 1989). Outre la confiance des clients, les mécaniciens bénéficient de la reconnaissance en tant qu'interlocuteurs privilégiés par les autres acteurs liés à la réparation : les vendeurs de pièces détachées et des casses auto leur proposent les «tarifs professionnels »; plus d'un opérateur de contrôle technique ferme l'œil sur les voitures qui lui sont amenées tout en faisant « passer » les tests ; les garagistes leur adressent certains clients pour des interventions spécifiques ou pour accélérer les délais. Auprès de ses contacts, le mécanicien de rue se présente comme l'homme à tout faire aux «mains en or » et capable d'avoir les bons «tuyaux». Son habilité se démontre dans son rôle d'intermédiaire et de consultant pour ses clients.

Cette reconnaissance professionnelle est entretenue par les mécaniciens eux-mêmes qui ne lésinent pas pour colporter les histoires de réparations difficiles « résolues » ainsi que pour souligner les exploits réussis dans des conditions particulières (Orr, 1990). Leur autorité et compétence en matière de réparation se base en effet sur un patrimoine oral composé de récits, d'histoires, de performances et d'avis formulés et diffusés grâce au «bouche à oreille » entre clientes et autres professionnels. Pour le mécanicien de rue, se voir reconnu en tant que professionnel, sans posséder la moindre licence, demeure important pour ses activités et aussi pour alimenter son orgueil personnel et son sentiment de réalisation professionnelle (Crawford, 2009). À plusieurs reprises, les mécaniciens que j'ai suivis ont « reconnu » des voitures qu'ils avaient déjà réparées en formulant appréciations ou conseils sur leur manutention. La réalisation de contrôles périodiques sur les mêmes voitures, à l'imminence des vacances ou des contrôles techniques, permet au mécanicien de valoriser et de conserver dans le temps sa compétence experte. L'orgueil de «suivre » un véhicule est un moyen pour fidéliser

\footnotetext{
${ }^{7}$ Exemple issu du carnet de terrain (07/04/2011): «Arnaud [un client] fait l'éloge plusieurs fois de Mel pour son habileté ; en lui tapant sur l'épaule s'exclame : «Tu sais, au garage ils m'ont demandé 80 balles pour changer un amortisseur... toi tu fais ça pour beaucoup moins cher... et bien! ».
} 
le client et aussi pour réduire un peu les risques de retravailler sur des réparations rafistolées d'autres mécaniciens de rue. Mel détaille souvent des bonnes pratiques à suivre pour la manutention de la voiture et les « combines » pour passer le contrôle technique, comme dans cet extrait du carnet de terrain (18/06/2011):

Tu me disais que ça fait un moment que tu ne roules pas, non ? Alors je t'explique, avant de venir avec moi à faire le contrôle tu conduis pour une bonne heure en poussant à fond toutes les vitesses. [...] Tu vas voir on va gagner au niveau pollution.

La capacité de répondre aux défis occasionnés par les réparations dans la rue s'accompagne de la volonté de se dire et de se faire reconnaître en tant qu'expert. Cette reconnaissance est une source d'identification en tant que professionnel de la réparation : lors des échanges avec les clients c'est aussi la réputation des mécaniciens qui est en jeu. On fait ici référence à une dimension plus large que la simple satisfaction professionnelle car les mécaniciens agissent dans les lieux où se trouve leur entourage de référence. Par sa visibilité, ses nuisances et ses opportunités, leur activité s'inscrit dans un environnement de proximité spatiale et sociale. Les voisins et les riverains établissent un rapport personnel avec le mécanicien au-delà des seules sollicitations pour des réparations. Il peut devenir une personne digne de confiance, à qui demander un prêt d'outils ou des services variés 8 . Ainsi, les mécaniciens agissent comme acteurs dans le terrain de leur quartier en créant des liens sociaux entre riverains, en s'occupant des petites réparations ainsi que de la sauvegarde des espaces communs. La bonne réputation de Mel est perceptible dans les salutations affectueuses de ses voisins ; il s'est d'ailleurs occupé de réparer les sonnettes d'un bâtiment de son HLM et se charge de prévenir les enfants qui jouent dans la même cour où il répare les voitures lors d'un danger. À partir de son activité de travail, le mécanicien de rue peut trouver sa place dans un écosystème de relations sociales ancrées dans les quartiers populaires, où il agit et où il est reconnu autant par sa compétence que par son rôle social.

\section{Conclusion}

Les activités du mécanicien de rue donnent à voir une image qui a une double dimension: ses compétences et ses qualités dans l'art de la débrouille d'un côté, les multiples contraintes auxquelles il est exposé pendant son travail de l'autre côté. Bien que son action soit marquée par une précarité existentielle (Cingolani, 1986), son inscription dans un tissu social également touché par de fortes incertitudes et difficultés de revenu lui permet d'accéder à des sources de satisfactions et de reconnaissance de son rôle professionnel et social (Laé, 1989). Cette figure sociale est

\footnotetext{
${ }^{8}$ La confiance d'autrui n'est toutefois jamais donnée : un exemple est l'histoire de Camara, un mécanicien de rue qui a dû fuir son quartier suite à de nombreux épisodes problématiques lors des réparations ainsi que dans ses rapports avec les riverains de son lieu habituel de réparation.
} 
prise entre, d'un côté, l'assujettissement aux incertitudes et, de l'autre, une subjectivation valorisée en tant qu'expert dans la réparation, même s'il occupe une place marginale dans la hiérarchie sociale de ces professionnels (Lhuilier, 2005). En conclusion, je veux souligner trois aspects marquant de cette situation.

Premièrement, l'incertitude fait partie de l'horizon quotidien des mécaniciens de rue : ils sont toujours confrontés aux risques, aux imprévus et aux limites. Pour y faire face, ils développent des services spécifiques et des réparations qui sont le résultat de leur posture de bricoleurs, tout en répondant ainsi aux demandes de leur clientèle. Ces pratiques de « débrouille créative » se montrent capables de (re)composer les diverses ressources mobilisables pour résoudre les innombrables imprévus (Latouche, 1991). Cette forme d'action indique une démarche "tactique» selon la conception de de Certeau (1990). La mise en perspective de ces pratiques comme actions tactiques, permet de restituer tant la composante créative au cœur du métier des mécaniciens de rue, que la profonde condition de précarité dans laquelle ils évoluent. Les pratiques du mécanicien de rue peuvent être considérées comme un art des faibles dans la mesure où elles se déploient dans un temps compressé et limité faute de pouvoir s'exprimer dans un lieu stable (de Certeau, 1990 : 63). Ces pratiques et les solutions créatives, adoptées tout au long du travail, ne leur permettent toutefois pas de s'émanciper de la situation d'incertitude dans laquelle ils agissent et à laquelle ils sont assujettis.

Deuxièmement, pour réaliser leurs services, les mécaniciens mûrissent une connaissance sensible (Gibson, 1979; Goodwin, 1995) capable de permettre l'appréciation du « travail bien fait » lors des réparations, mais aussi de cumuler une expérience grâce aux réparations réussies (Sudnow, 1978). La simple réalisation des réparations et des services dans un contexte aussi précaire n'empêche pas, mais au contraire soutient la construction d'une réputation en tant qu'expert et professionnel dans la réparation. Le mécanicien exprime ainsi sa responsabilité lors des services fournis et peut bénéficier par-là de la confiance attribuée avant tout par les clients et les riverains. Ce processus contribue à la construction de son identité professionnelle et, par ce biais, influence sa subjectivation comme individu actif et reconnu dans son entourage social, ainsi que dans le quartier où il travaille et souvent habite. Son activité de travail, en plus de lui apporter un revenu, peut être reconnue comme utile pour ceux qui habitent là où il agit. Sa figure, quand il arrive à se faire accepter et à maintenir son capital-réputation (Bevort, Lallement, 2006), peut donc se configurer comme une ressource du quartier, en termes de savoir et de savoir-faire ainsi qu'en tant qu'intermédiaire réputé et recherché, comme l'avait déjà souligné Sudhir Venkatesh (2006) dans sa description des ghettos américains et le collectif Rosa Bonheur (2017) à propos de la banlieue lilloise.

Troisièmement, les pratiques du mécanicien, éloignées des formes les plus « simples » ou « traditionnelles » de travail (subordonné, d'exécution et répétitif) souvent associées au travail informel, se montrent plus proches des figures liées à l'entrepreneur de soi (Gorz, 2001). Le mécanicien de rue, avec la multiplicité de services qu'il propose et les 
risques qu'il doit maîtriser au quotidien, apparaît comme un travailleur flexible, précaire et voué à la multiactivité (Boltanski, Chiapello, 1999 ; Cingolani, 2014). À partir d'un regard orienté sur une activité inscrite dans l'économie informelle, on peut ainsi noter des similitudes avec d'autres formes de travail caractéristiques de la situation contemporaine. La figure du mécanicien peut donc être considérée comme celle d'un "entrepreneur de soi-même» qui agit dans le contexte de quartiers populaires (Tafferant, 2007) et doit en permanence s'activer pour maintenir sa clientèle et sa place. Le cas des mécaniciens peut aussi souligner la forte ambivalence associée aux compétences d'autonomie, de créativité et de responsabilité. Ces dernières sont souvent célébrées comme des éléments associés à la liberté du travailleur (Bologna, Fumagalli, 1997) et aux métiers les plus prestigieux. Mobiliser et maîtriser ces compétences, indispensables aussi pour la réalisation d'activités très précaires, n'émancipe pourtant pas les travailleurs des contraintes qui leur sont imposées (Ross, 2009).

\section{Bibliographie}

ARMANO E., Murgia A. (dir.) (2012), Mappe della precarietà. Knowledge workers, creatività, saperi e dispositivi di soggettivazione, Bologne, Odoya.

BASTIEN S. (2007), «Observation participante ou participation observante ? Usages et justifications de la notion de participation observante en sciences sociales ", Recherches qualitatives, vol. 27, n 1, p. 127-140.

BERARDI F. (2016), L'anima al lavoro. Alienazione, estraneità, autonomia, Rome, Derive Approdi.

BeVort A., LALLEMENT M. (2006), Le capital social : performance, équité et réciprocité, Paris, La Découverte.

Bologna S., Fumagalli A. (1997), Il lavoro autonomo di seconda generazione, Rome, Derive Approdi.

Boltanski L., ChiaPello E. (2000), Le nouvel esprit du capitalisme, Paris, Gallimard.

Bouffartigue P., Busso M. (2009), «Précarité, informalité: une perspective Nord-Sud pour penser les dynamiques des mondes du travail », Les mondes $d u$ travail, $\mathrm{n}^{\circ} 9-10$, p. 27-40.

Bruni A., GHERARDi S. (2007), Studiare le pratiche lavorative, Bologne, Il Mulino.

CASTEL O. (2007) « De l'économie informelle à l'économie populaire solidaire : concepts et pratiques », in F. HAINARD, O. CREVOSIER, P. ISCHER (dir.), L'économie informelle : une alternative à l'exclusion économique et sociale ?, Berne, CNSU, p. 111-134.

Clerval A. (2016), Paris sans le peuple : la gentrification de la capitale, Paris, La Découverte. 
Cingolani P. (1986), L'exil du précaire, Paris, Méridiens-Klincksieck.

Cingolani P. (2006), La précarité, Paris, PUF.

Cingolani P. (2014), Révolutions précaires : essai sur l'avenir de l'émancipation, Paris, La Découverte.

Collectif Rosa Bonheur (2014), « Comment étudier les classes populaires aujourd’hui ? Une démarche d'ethnographie comparée», Espaces et sociétés, vol.156-157, nº1, p. 125-141.

COLLECTIF RosA BONHEUR (2017), «Les garages à ciel ouvert : configurations sociales et spatiales d'un travail informel », Actes de la recherche en sciences sociales, vol. 216-217, $\mathrm{n}^{\circ} 1$, p. 80-103.

Coletto D. (2010), The informal economy and employment in Brazil: Latin America, modernization, and social changes, New York, Palgrave Macmillan.

Corbin J., Strauss A. (1993) "The articulation of work through interaction», The sociological quarterly, vol. $34, \mathrm{n}^{\circ} 1$, p. $71-83$.

CRAWFORD M. B. (2009), Shop class as soulcraft: an inquiry into the value of work, New York, Penguin.

DE Certeau M. (1990 [1980]), L'invention du quotidien : Arts de faire, Paris, Folio.

Fol S. (2010), «Mobilité et ancrage dans les quartiers pauvres : les ressources de la proximité », Regards sociologiques, $\mathrm{n}^{\circ} 40, \mathrm{p} .27-43$.

GARFINKEL H. (1963), «A conception of and experiments with trust as a condition of concerted stable actions », in J. O'BRIEN (dir.) (2006), The production of reality: essays and readings on social interaction, Londres, Sage, p. 381-392.

GiBSON J. (1979), The ecological approach to visual perception, Boston, Houghton Mifflin.

GioRdano D. (2016), «Conflits et arrangements dans les parkings de banlieue: les pratiques des mécaniciens de rue », in J. Calderon, L. Demally, S. Müller (dir.), Aux marges du travail, Toulouse, Octares.

Goodwin C. (1995), « Seeing in depth », Social Studies of Science, vol. 25, n 2, p. 237-274.

Gorz A. (2001), «La personne devient une entreprise », Revue du MAUSS, vol. 18, $\mathrm{n}^{\circ} 2$, p. 61-66.

Hughes E. (1951), « Work and the self », in J. H. ROHRER, M. SHERIF (dir.), Social psychology at the crossroads, Oxford, Harper, p. 313-323.

Hughes E. (1959), "Prestige», Annals of the American Academy of Political Sciences, vol. $325, n^{\circ} 1$, p. $45-49$. 
Jounin N. (2008), Chantier interdit au public : enquête parmi les travailleurs du bâtiment, Paris, La Découverte.

LAE J.-F. (1989), Travailler au noir, Paris, Metaillé.

LATOUCHe S. (1991), La planète des naufragés: essai sur l'après-développement, Paris, La Découverte.

LeVI-STRauss C. (1961), La pensée sauvage, Paris, Plon.

LHUILIER D. (2005), « Le « sale boulot », Travailler, vol. 14, n², p. 73-98.

ORR J. (1990), «Sharing knowledge, celebrating identity: war stories and community memory in a service culture ", in D. Middleton, D. Edwards (dir.), Collective remembering: memory in society, Beverley Hills, Sage.

Pillon T. (2012), Le corps à l'ouvrage, Paris, Stock.

PINK S. (2009), Doing sensory ethnography, Londres, Sage.

Portes A., Castells M., Benton L. (1989) (dir.), The informal economy. Studies in advanced and less developed countries, Baltimore, Johns Hopkins University Press.

Ross A. (2009), Nice work if you can get it. Life and labour in precarious times, New York, New York University Press.

Schatzki T. R., Knorr Cetina K., von Savigny E. (2001) (dir.), The practice turn in contemporary theory, New York, Routledge.

SASSEN S. (2001), The global city: New York, London, Tokyo, Princeton, Princeton University Press.

Sennett R. (2008), The craftsman, Yale, University Press.

Standing G. (2011), The Precariat. The new dangerous class, Londres, Bloomsbury.

SuDNOw D. (1978), Ways of the hand. The organization of improvised conduct, Cambridge, Harvard University Press.

TAFFERANT N. (2007), Le « Bizness » : une économie souterraine, Paris, PUF.

VenKatesh S. (2006), Off the books. The underground economy of the urban poor, Cambridge, Harvard University Press.

WACQUANT L. (2002), Corps et âme: carnets ethnographiques d'un apprenti boxeur, Marseille, Agone.

WEBER F. (1989), Le travail à-côté. Une ethnographie des perceptions, Paris, EHESS.

Williams C. (2013), «Out of the shadows: a classification of economies by the size and character of their informal sector », Work, Employment er Society, vol. 28, n 5, p. 1-19. 